\title{
The Role of Equilibration in Piaget's Theory of Cognitive Development and Its Implication for Receptive Skills: A Theoretical Study
}

\author{
Hamidreza Babaee Bormanaki \\ Department of English Language and Literature, University of Guilan, Rasht, Iran \\ Yasin Khoshhal \\ Department of English Language and Literature, University of Guilan, Rasht, Iran
}

\begin{abstract}
Piaget's theory, which is at the center of cognitive approaches to psychology and learning, gave a significant importance to the construct of equilibration. This construct plays a paramount role in cognitive adaptation. It is the unifying factor, and thus the sufficient condition of cognitive development. An attempt was made in this paper to revisit the construct of equilibrium in the realm of cognitive development and relate it to the two receptive language skills, namely; listening and reading comprehension. Topics analyzed included, overview of Piaget's theory of cognitive development, assimilation and accommodation in the process of equilibration and their role in cognitive adaptation, kinds of equilibration, schema theory, Rutherford's neoPiagetian view of assimilation and accommodation. Then the process of equilibration was related to the practical contexts and its implications and contributions to the listening and reading comprehension was described. It's also described how critical thinking, schemata, assimilation, accommodation, and reading comprehension are interrelated to each other in implication section. Finally, Rutherford's neo- Piagetian view is contributed to reading comprehension from socio-cultural perspective.
\end{abstract}

Index Terms-Piaget's theory of cognitive development, assimilation and accommodation, equilibration, schemata, Rutherford's Neo-Piagetian view

\section{INTRODUCTION}

The term development in its broadest psychological perspective denotes to definite transformations that happen in human beings (or animals) amid the outset and closing stages of their life. The term is not applied to all transformations, but rather those that emerge in systematic ways and hang about for a rationally long period of time. Psychologists also make a value judgment in determining which changes qualify as development. The changes, at least those that occur early in life, are generally assumed to be the better and to result in behavior that is more adaptive, more organized, more effective, and more complex (Mussen, Conger, \& Kagan, 1984, as cited in Woolfolk, Winne and Perry 2003).

According to Woolfolk, Winne and Perry (2003), human development can be defined into a number of different perspectives. Physical development, which is associated with body, Personal development deals with the changes in individual's personality. Social development takes into account the relationship between people and tries to define change in this way. Cognitive development as the name infer, identifies change in thinking and cognitive realms of human being (p. 24).

Although there is disagreement about the way of the development or what is involved during the development, there are some general principles that theorists in this domain would agree with. These principles are; "1. People develop at different rate. 2. Development is relatively orderly. 3. Development takes place gradually" (Woolfolk, Winne \& Perry, 2003 , p. 25). Based on the first principle, we can see many students in our classrooms in which, some of them are tall and some of them are short or some of them are intellectually better than others and the others are slow at the rate of their learning or some students are social and make relationship with their fellow students but conversely the others are embarrassed and silent. To concretize the second principle, we can see that the children first sit before they can walk or babble before they can talk. The third principle states that, development is not happened at one time; instead it takes place gradually and for a long period of time, provided that some preconditions are shaped and constructed for the next time of the development.

Theories of development provide a framework for thinking about human growth, development, and learning. Understanding these theories can provide useful insight into both the individual and societal influences on early development (Armstrong et al 2014). Over the last decade's various theories of early child development have been proposed. Although these theories have been significant in their own right, they have also had some limitations. Armstrong et al (2014) enumerated and incorporated some of these theories precisely and highlighted them which help to explain how children learn and grow, by what means their behavior may be modified and improved, and why it is argued that their outcomes are shaped by both biology and experiences. These theories are; attachment theory which 
emphasizes the development that is the result of the relationship between children and their parent. Cognitive theory that is concerned with children's cognitive development, attributed to psychologist Jean Piaget. The focal point of this theory is that, cognitive skills progress through a series of stages in which new information from experiences is taken in and understood. Ecological Systems Theory which helps to explain how children develop within the context of their world. In this theory, there are five systems that influence development; Microsystem, Mesosystem, Exosystem, Macrosystem, and Chronosystem, and considered that the person's biology also contributed to these systems. Prevention Model which emphasizes multiple layers of supports and services aimed to decrease risk factors and reduce disorders, in order to promote better outcomes. Parenting Styles. It refers to the degree to which parents respond to their child's needs, disciplinary strategies they use, parental expectations for maturity and control, and the effects that this has on their child's development (p. 22-28).

As we saw, there are various models and theories which are influential and notable but researches have shown that Piaget's theory has been more influential than others. In this theory Piaget introduces the construct of equilibration. This construct or process has a paramount role in his theory and a necessary condition for cognitive adaptation which lead the child to the cognitive development. According to Piaget (1952, as cited in Aloqaili, A.S., 2011) equilibrium is a balance between two other processes namely; assimilation and accommodation. According to Woolfolk, Winne and Perry (2003), Piaget also introduces other important construct, named schema to the realm of psychology and education. In fact, schema or (pl. schemata) plays a significant role in the process of assimilation and accommodation. This article is dealt with Piaget's construct of equilibration, its role in cognitive development and its implication for two language receptive skills namely, listening and reading. In this article, I try to discuss and investigate this construct from educational perspective in order to associate it with listening and reading comprehension.

\section{Piaget's Theory of Cognitive DeVelopment}

The most influential theorist in the study of cognitive development was Jean Piaget, who was born in 1896 and died in 1980. His prolific career in psychology spanned an astonishing 7 decades. Jean Piaget was no ordinary child. From a very early age, he showed tremendous intellectual talent and even from the age 15 to age 18 published various influential articles. His insightful descriptions of children's thinking changed the way we understand cognitive development. (Cook \& Cook, 2005).

According to Piaget (1954, as cited in Woolfolk, Winne and Perry 2003), certain ways of thinking that are quite simple for an adult are not simple for a child. Sometime all we need to do to teach a new concept is to give a student a few basic facts as background. At other times, however, all the background facts in the world are useless. The student is not simply ready to learn the concept. As we can infer, providing a few basic facts is not all the child need for his cognitive development. There are other factors that would interact to influence change in thinking. Piaget (1970, as cited in Woolfolk, Winne and Perry 2003), identified these factor and nominated them as; biological maturation, activity, social experiences, and equilibration. At first I concretize and characterize the first three factors and then I will return to the equilibrium factor in the next section and will discuss it precisely.

According to Woolfolk, Winne and Perry (2003) Maturation is the one of the important pre-requisite for the way that we make sense of the world. This factor is dealt with biological changes and unfolds these changes that are programmed in each human being at conception. Parents and teachers have little impact on this kind of change except in the case of the provision of healthier conditions which are necessary for maturation. Second factor, activity relate physical maturation to the environment and assumes that, over time whenever physical ability gains its maturation, its acts upon environment and learn from it. This factor deals with concepts such as observation, evaluation, and hypothesis formation. Children through these concepts, in other words through their physical maturation discover their milieu. The third factor is social experiences. Paget identified this factor by taking the social context into consideration. According to this factor we cannot neglect social context, social transmissions and the relationship between people, which all influence cognitive development. We can learn from our context and culture by social transmissions and this learning is largely depended on the individual's current stage of cognitive development.

\section{A. Two Basic Inclinations and Tendencies in Thinking}

According to Piaget's research on biology (as cited in Woolfolk, Winne and Perry 2003), human beings have two basic tendencies. The first one is the tendency toward organization which deals with the combining, arranging, recombining, and rearranging of the behavior and thought in the coherent system. The second tendency is toward adaptation which is concerned with adapting and adjusting with the environment.

\section{Organization}

This concept assumes that people have a tendency to organize their thinking processes into psychological structures. These psychological structures help us to understand and interact with the world. In this way, simple structure combined together to become more effective and sophisticated. Very young infants, for example, can either look at an object or grasp it when it comes in contract with their hands. They cannot coordinate looking and grasping at the same time. As they develop, however infants organize these two separate behavioral structures into a coordinated higher level structure of looking at, reading for, and grasping the object. They can of course, still use each structure separately (Ginsburg \& Opper, 1998; Miller, 1993, as cited in Woolfolk, Winne and Perry 2003). 
The important concept which requires special attention is schema (pl. schemata). In Piaget's theory this concept has its own place and for him, this concept is very important in the process of cognitive development. Schemata are the networks of structures in our mind and help us to encounter and make sense of the milieu around us. They are very specific, for example, from eating an ice cream schema to taking part in a party. So when we are encountered with the milieu and environments, related schemes are activated and allow us to make sense from our environment. According to Piaget (1970, as cited in Woolfolk, Winne and Perry 2003) as persons thinking processes become more organized and sophisticated, new schemes develop and behavior also become more sophisticated and better suited in the environment. Over the last years this concept has been extended to some disciplines related to education especially in the domain of language teaching and learning to include teaching and learning speaking and listening skills accordingly. I will return to this notion and its implication for the realm of language teaching and learning in implication section.

\section{Adaptation}

As the term suggests this concept refers to the human being's adjustment to the new environment. It can be defined as "changing one's cognitive structure or one's environment (or both to some degree) in order to better understand the environment" (Cook \& Cook, 2005, p. 5-7). According to the Piaget, in the process of adaptation or adjustment two basic processes are involved, namely; assimilation and accommodation. These two basic processes or in other words, pre-requisites are important for adaptation to take place.

\section{B. Assimilation}

According to Pritchard and Wollard (2010) assimilation is the collecting and classifying of new information. Schema has a paramount importance in this process and this is a notional representation of what an individual knows (or can do) and consists of discrete items of knowledge which are linked to each other by the common theme of the schema. When new information is encountered, for example, a car with no roof, a car painted with flowers, this is added to the existing schema; in other words, it is assimilated. However, it will only be assimilated if it does not contradict something already established as an integral part of what exists (p. 12). In this process, we may have to distort the new information to make it fit. For example; the first time many children see a skunk, they call it a "kitty." In this process they try to match the new experiences with an existing schema for identifying animals (Woolfolk, Winne, \& Perry, 2003).

As we can infer from this example, scheme plays a paramount role in the process of assimilation and without which the process encounter with some of the problems. Sometimes the existing schemata are not in accordance with incoming information, so in this situation, the process will be changed to accommodate new information in the process of accommodation.

\section{Accommodation}

"Accommodation refers to the process of changing internal structures of knowledge in order to have consistency with external reality" (Yang, 2010, p. 203). In the process of accommodation existing schemata should be changed to accommodate and include incoming and new information. In this process we modify old schemes or create new ones to better fit assimilated information. (Cook \& Cook, 2005). For example, sucking through straw may require some accommodation if the straw is of different size or length than the type we are accustomed to. There are also times when neither assimilation nor accommodation is used. For example, when we encounter something that is very unfamiliar, we actually ignore it (Woolfolk, Winne, \& Perry, 2003). For example, in listening to the conversation when we haven't had any experience and knowledge of the language of that conversation. So in this situation we probably ignore the conversation and prefer not to use the processes of assimilation and accommodation.

To make a distinction between assimilation and accommodation, Wadsworth (1996 as cited in Aloqaili, A.S., 2011), claims that accommodation deals with development (a qualitative change) and assimilation deals with growth (a quantitative change); together these processes account for intellectual adaptation and the development of intellectual structures. On the other hand, Rubin (1997 as cited in Aloqaili, A.S., 2011) asserts that, in spite of the importance of both assimilation and accommodation as a cognitive process development, children should be aware of making balance between these two processes. Therefore, balancing between assimilation and accommodation is the function of the third cognitive mechanism, which is equilibrium. A brief explanation of equilibrium is provided below.

\section{Equilibration}

Equilibration is one of the hypothetical constructs used by Piaget to explain part of his theory of cognitive development. As the name suggest, the process of equilibration requires the balance between assimilation and accommodation. According to Gallagher (1977 as cited in Fajemidagra) equilibrium is the core of Piaget's theory of cognitive development which must be well perused. Piaget (1977 as cited in Fajemidagra) contends that equilibration should be regarded as a process, the search for a better state of equilibrium. The type of equilibrium state which Piaget refers to is not the physical type but a psychological equilibrium state. According to Flavel (1963 as cited in Fajemidagra) equilibrium states are composed or organized systems of actions (e.g. sensory motor, perceptual and concrete operations) whose attributes as systems are describable in equilibrium terms. The use of the term "equilibration" as self-regulation or auto regulation stems from Piaget's constant focus on the analogy between biological and cognitive adaptation. Admittedly, both of which require the two poles of assimilation and accommodation tending toward harmony through successive equilibrations (Piaget \& Inhelder, 1969 as cited in 
Fajemidagra). According to Piaget (1964 as cited in Fajemidagra) equilibration is an active and dynamic process. It is a process of self-regulation. It is a model of an equilibrated "system" where a transformation in one direction is compensated for by a transformation in the other direction. Kaiplus and his colleagues (1977 as cited in Fajemidagra) have attempted to operationally define equilibration within the realm of learning and instruction in science. They explain that reflection, restructuring of tasks, and learning from others contributes to the formation of new reasoning patterns.

\section{Kinds of equilibrium}

According to Piaget (1977 as cited in Fajemidagra) the first type of equilibrium pertains to the relationship between assimilation and accommodation. Before this type of equilibrium can be attained, there should be a balance between the cognitive structures of the subject and the objects. The subject's cognitive structures accommodate to the new object being presented and at the same time assimilate the object into his structures. The second kind of equilibrium is equilibrium among the subsystems of the subject's schema. In reality, the schemas of assimilation are coordinated into partial systems, which are referred to as subsystems in relation to the totality of the subject's knowledge. The subsystems can present conflicts among themselves. This type of conflict is illustrated by Piaget (1977, p. 11 as cited in Fajemidagra) by citing the logic mathematical operations. He claims that it is possible to have conflicts between a subsystem dealing with classifications, seriation, and number construction. The third kind of equilibrium in cognitive development is determined by the balance between the parts of the subject's knowledge and the totality of his knowledge at any given moment. Piaget labeled this type of equilibrium as "fundamental." He argues that often there is a constant differentiation of the totality of knowledge into parts and an integration of the parts back into the whole. Before knowledge can develop, there must be a sort of coordination between the two processes. In cognition, an attempt to integrate back what has been differentiated poses new problems. 'These new problems lead to the construction of new actions upon the previous actions or new operations upon the previous actions or new operations upon the previous operations. The construction of operations upon operations presumably, may enhance transition from one stage to the next." (Piaget 1977, p. 12 as cited in Fajemidagra).

Regardless of the kinds of equilibrium that are spanned by the equilibration process, Flavell, (1977, p. 242 as cited in Fajemidagra) enumerated the following four things which a child needs the ability or disposition to do in sequence before equilibration can set in.

1. Attend to or notice both of the apparently conflicting elements of a situation.

2. Interpret and appreciate these elements as conflicting and, therefore problematic - a young child may not be able to do this,

3. Respond to the sensed conflict by progressing rather than regressing, e.g., by trying to explain it rather than by clinging defensively to his initial belief or refusing to have anything more to do with the problem.

4. Come up with a better conceptualization of the situation that can resolve the apparent conflict and thereby "reequilibrate" his mental structure at a higher development level.

\section{The process of equilibrium}

To concretize and put simply the whole discussion of the equilibration stated above, we can say that, the developing mind is constantly seeking an equilibration; the balance between what is known (the current schemata) and what is currently being experienced (the incoming information and new knowledge). This balance is accomplished by two subprocesses of assimilation and accommodation. Working in conjunction with each other, these two sub-processes construct the cognitive adaptation by the whole processes of equilibration. (Williams \& Burden, 2000).

It is worth noting that sometimes schemata do not produce a satisfying result and this situation lead to the disequilibrium and we become uncomfortable. This motivate us to keep searching for a solution through assimilation and accommodation, and thus our thinking changes and move ahead until the incoming information accommodated and we make sense of the new information. (Woolfolk, Winne, \& Perry, 2003). "To resolve the disequilibrium, we accommodate, or adjust, our schemes to provide a better fit for the new experience. If we are successful, we achieve cognitive equilibrium. Equilibration therefore is the dynamic process of moving between states of cognitive disequilibrium and equilibrium as we assimilate new experiences and accommodate schemes." (Cook \& Cook, 2005, p. 5-8)

To delineate and concretize the whole process of the cognitive adaptation, let's have an example from (Cook \& Cook, 2005):

"Lily, a 2-year-old who is learning to name animals, (the process shown in Figure 5.1). Lily has a dog at home, and according to her "doggie scheme," "doggies" are animals that have four feet and fur and that bark and fetch balls. One day, riding in the car with her mother, Lily points to a field with several cows and exclaims, "Look, Mommy, doggies!" She is excited to see so many "doggies," especially ones so large! We can see that Lily is trying to understand these new animals by thinking about them as something she already understands: "doggies." This is an example of assimilation, the process of bringing new objects or information into a scheme that already exists. Thinking of these new animals as "doggies," Lily fully expects that they will also bark and fetch balls. Her mother, however, comments, "No, those are cows. They are bigger than dogs. And see the udders underneath? Cows give us milk." These comments place Lily into cognitive disequilibrium — she is confused. Lily realizes that she has never seen udders under dogs and also has never seen dogs that large. To resolve her cognitive conflict, Lily adjusts her understanding of 
animals. She adds new information about dogs (they are smaller and don't give us milk), and she learns a new animal (cows are like dogs but larger, and they give milk). These adjustments are examples of accommodation Now Lily can properly identify dogs and cows, and her new success in naming the animals moves her into cognitive equilibrium" (Cook \& Cook, 2005, p. 5-7).

The fallowing diagrammatic representation at the next page delineates the example appropriately:
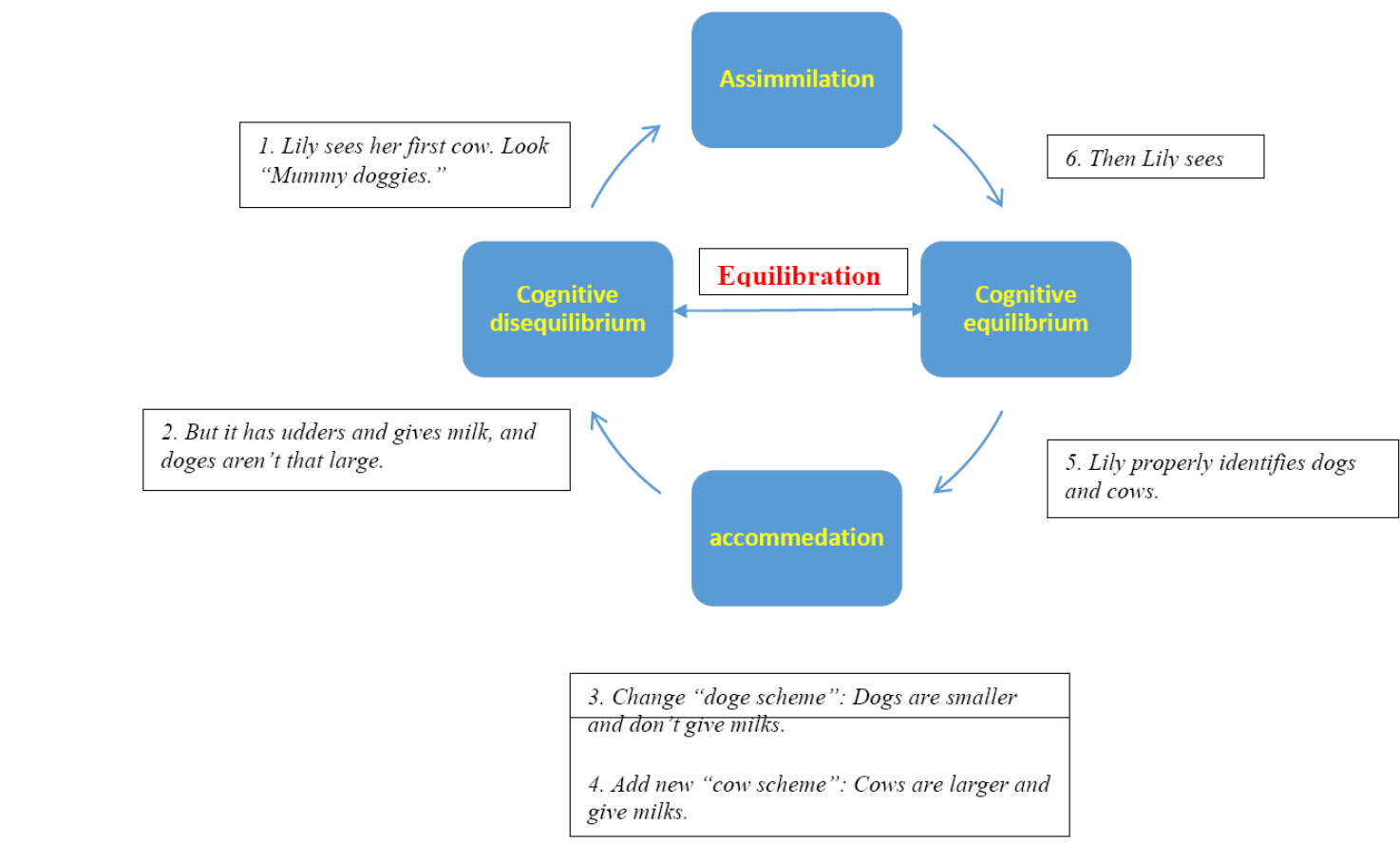

Figure 1. Adaptation and Equilibration

In the cycle of adaptation and equilibration, a new experience is first assimilated into an existing scheme. If it doesn 't fit properly, cognitive disequilibrium results. Accommodating (adjusting) the scheme brings the child to cognitive equilibrium, until a new assimilat ion challenges the scheme again (Cook \& Cook, 2005, p. 5-7).

\section{Neo-Piagetian Model of Assimilation And Accommodation in the Cognitive (Structural) AND Cultural (BeHAVioral) ReAlms}

Since Piaget's theory of equilibrium, there has been a lot of interest about this basic concept and how to put this process into practice in educational institutions and especially in the process of teaching and learning. On the other hand, since one of the key requisites of this process was social relationship, so this construct or process has been extended to take into account the social cultural context. One of the researchers who have related the theory of equilibrium to the cultural context is Rutherford (2011). He has constructed the model of assimilation and accommodation in the cognitive and cultural realms. He has defined cognitive assimilation as construct growth for internal system's fit, and cognitive accommodation as construct change for external/environmental fit, cultural assimilation as prevailing culture growth as diverse cultural identities/groups adapt and adjust for contextual fit and cultural accommodation as prevailing culture change to fit plural cultural group's/identities" traditional practices and beliefs. In his new model, the "Me" - "I" cognitive and "Us" - "We" cultural dynamics are employed for grounding and delineating the model and neo-Piagetian developmental sequences are expounded separately for the "Me" (assimilatory cognitive), "I" (accommodatory cognitive), "Us" n (assimilatory cultural) and "We" (accommodatory cultural) realms (p. 1).

To ground the model, the writer has applied the systems-theoretic dimensions of "ego, system" and "cult, system", for example, in partnership with the contextual constructs of "environment, ego" and "environment, cult" (see the following figure for parallel system-context relations). Relative to these dimensions, he also argues that a "Mepathway", and "I-pathway" are social-psychological products of the cognitive processes of assimilation and accommodation, respectively. Correspondingly and also relative to these dimensions, He also argues for an "Uspathway", and "We-pathway" which are social-psychological products of the cultural processes of assimilation and accommodation, respectively. Following figure from Rutherford (2011) depicts these systems-based relationships: 


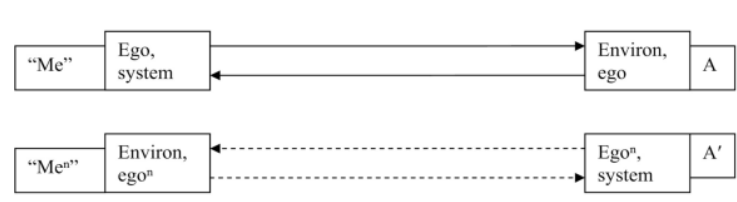

[D] \& [D]: Accommodation in the Cultural realm.

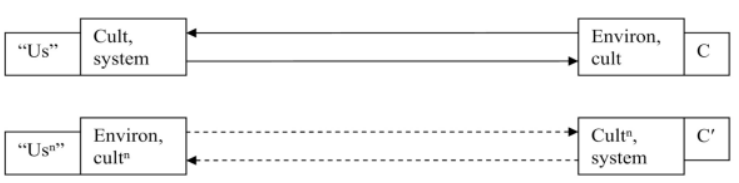

[B] \& [B']: Accommodation in the Cognitive realm.
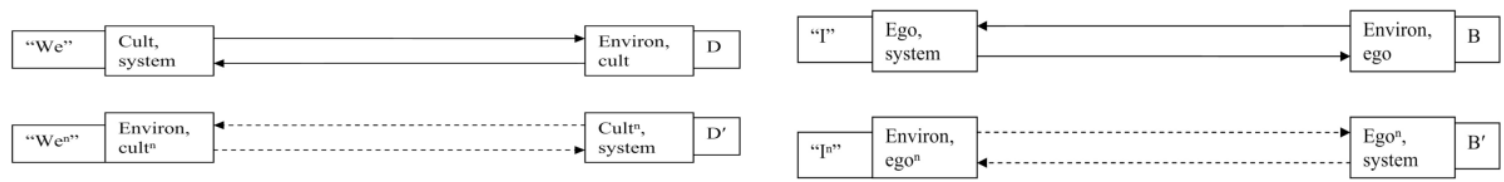

Figure 2. Neo-Piagetian model of Assimilation and Accommodation in the Cognitive (structural) and Cultural (behavioral) realms (Rutherford, 2011).

According to Rutherford, we can understand from the diagram that:

"Cognitive Assimilation - entails the process whereby an individual's constructs grow from interaction with the environment, for internal fit

Cognitive Accommodation - entails the process whereby an individual's constructs change through interaction with an environment, for external fit

Cultural Assimilation - entails the process whereby a prevailing context/culture grows as diverse cultural identities/groups adapt (intelligently) and adjust (personally) to "fit in", for external fit (\& unity)

Cultural Accommodation - entails the process whereby a prevailing culture/context changes through plural cultural identities/groups living out their traditional beliefs and practices, for internal fit (\& diversity)" (Rutherford, 2011, p. 2).

To delineate the above figure, Rutherford (2011) states that, [A] represents "Me" from a person's own (self/ego) side of the self-other interrelationship and $\left[\mathrm{A}^{\prime}\right]$ represents the "Me" ("Men) from an other's (ego) side. For instance, "Me" as a scientist and another "Me" as an artisan. [B] depicts the "I" from one's own (self/ego) side of the self-other interrelationship and $\left[\mathrm{B}^{\prime}\right]$ depicts the "I" ("In") from an other's (ego) side. For instance, "I" as an introvert and another "I" as an extrovert. [C] portrays the "Us" from one's own cultural group/identity's side of the sociocultural unit (cult, system) - culture (environment, cult) cross-cultural interrelationship and $\left[\mathrm{C}^{\prime}\right]$ portrays the "Us" ("Us") from another group's/cultural identity's side (cult ${ }^{n}$, system - environ, cult $^{n}$ ). For example, from the context of Australian national culture "Us" may be the Anglo-Celtic Australian community and another "Us" the Chinese Australian (or Australian Chinese) community. [D] illustrates the "We" from one's own cultural group/identity's side of the socio-cultural unit (cult, system) - culture (environment, cult) cross-cultural interrelationship and [D'] illustrates the "We" ("We"n") from another group's/cultural identity's side $\left(\right.$ cult $^{n}$, system - environ, cult $\left.^{n}\right)$. For example, from the context of world cities "We" may be Sydneysiders and another "We" Berliners (p. 3).

To conclude Rutherford's Neo-Piagetian model of Assimilation and Accommodation, it is worth noting that, he takes into account the socio-cultural dimensions of cognitive development and as a matter of fact this is the positive aspect of his theory. However, his model should be tested empirically for the purpose of validation and practical implication.

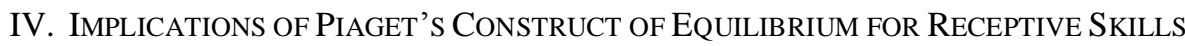

\section{A. Schema (Schemata)}

Piaget's construct of schema has been embraced by many of researchers in the realm of language teaching and learning over the last decades. In teaching the language skills, receptive skills, namely reading and listening have been related to this concept closely and schema plays an important role in teaching and learning of these two skills. One of the reasons of this relationship between schema and these skills is actually the practical inferences that can be drawn from the definition of this concept. Piaget (1977 as cited in Woolfolk, Winne, \& Perry, 2003) himself defines scheme as mental systems or categories of perception and experience that can be called whenever we encounter new situation or incoming information. This concept has been defined variously between researchers in the realm of cognitive psychology; however, the whole concept is common among these definitions. For example, Rumelhart (1984 as cited in Aloqaili, A.S., 2011) defined schema as an organized knowledge that one has about people, objects, places, events, processes, concepts, and virtually everything that provides a basis for learning. Bos and Anders (1990 as cited in Aloqaili, A.S., 2011) claimed that, schema theory describes how knowledge is structured in memory and how these structures affect incoming information. Anderson and Wilson (1986 as cited in Aloqaili, A.S., 2011) asserted that schema theory explains how people's existing knowledge affects comprehension. However, Over the last decades, this concept has been extended to include formal schema and content schema. According to Lynch (2006) "there are two 
kinds of schemata; content schemata and formal schemata. Content schemata are networks of knowledge on different topics, for example, "cooking," comprising knowledge gained from a range of sources and also personal experience. Formal schemata are derived from our knowledge of the structure of discourse genres, e.g., an academic lecture, a sermon" (p. 89).

\section{B. Schema and Listening Skill}

Schema has a paramount importance in listening skill. There are two types of processing related to the listening, namely bottom up and top down processing. The second one, top down processing is related to the theory of schema. In top-down processing we rely on what we already know to help make sense of what we hear; in other words, we rely on our schemata related to what we hear. In the case of content schemata as Lynch (2006) pointed out, when we hear someone talking about a topic that we are able to link to an existing content schema, then we find comprehension very much easier. On the other hand, in the case of formal schemata an awareness of what sort of discourse is being listened to, makes it easier to engage in top-down processing strategies, such as predicting and inferencing (p. 89).

To make a relationship between top down processing and the process of equilibration, it can be said that schemata plays an important role in the process of equilibration, in other words it balances the complex process of assimilation and accommodation and avoid the process of disequilibrium. For example, when we hear a speech with which we have the schema related to the topic of that speech, we can use the related schema to make sense of what we hear, in other words schema in this situation avoid us from the process of disequilibrium and enable us to reach to the cognitive adaptation.

\section{Schema and Reading Comprehension}

The theory of schema is tightly associated with reading comprehension. On applying this theory to reading, researchers (Grabe \& Swaffar, 1988) found that reading was an interactive process, i.e., it was a dynamic interaction between the writer and the reader in which the reader creates meaning from the text by activating his stored knowledge and extending it with the new information supplied by the text (Grabe \& Swaffar, 1988). This direction in reading research concentrated on the text-reader interaction. Indeed, that appears to be the current direction, with the added dimension of the social context, which came from the work of sociolinguists. This view to reading highlights the importance of sociocultural context for improving cognitive adaptation and avoiding cognitive disequilibrium. In my opinion, learners can rely on schemata in reading comprehension more than listening comprehension. The reason of this different dependence on schemata is laid down on the modality of these two skills. The modality of these skills is different. Modality of listening is sound; on the other hand, modality of reading is sight and visual motion. This modality makes speech transient, while this is not so matter in reading comprehension. Because reading is not transient, learners can rely on their schemata more than when they are listening to speech. So, this helps them to understand what they read and avoid them from disequilibrium. Readers also rely on the different kinds of schemata; namely content and formal schemata in their reading activity to better make sense of what they read and to reach the cognitive adaptation.

In the process of assimilation, Wadsworth (1996 as cited in Aloqaili, A. S. 2011) describes the assimilation process like adding air into a balloon and claims that, this process elaborates the size or growth of schemata, however, it does not alter them. He stated the following:

Assimilation theoretically does not result in a change of schemata, but it does affect the growth of schemata and is thus a part of development. One might compare a schema to a balloon and assimilation to putting more air in the balloon. The balloon gets larger (assimilation growth), but it does not change its shape. Assimilation is a part of the process by which the individual cognitively adapts to and organizes the environment'” (p. 17).

We can infer that assimilation has a paramount effect on the growth of schemata by adding or taking in new information to old. In the process of reading comprehension, our schematic knowledge can be enlarged to include the variety of concepts and topics by reading the variety of topics and materials. We can say that normal and gradual reading facilitate this process. Over time when we read more and more material, our schematic knowledge grows and grows by assimilating more and more concepts and incoming information and in a final run it helps us in the process of equilibrium. However, the assimilation process itself does not change or create new schemata. Change and creation of schemata are the functions of another cognitive development process, which is accommodation. Whenever we read material and incoming stimuli which are unfamiliar for us, in this situation we cannot assimilate new stimuli and we have to accommodate them, so, in this way we rely on two ways; to construct new schemata in which to place the stimulus, or change or modify the existing ones to fit with new stimulus. Rubin (1997 as cited Aloqaili, A. S. 2011) explains that a child (or a student) with equilibrium process would be able to see similarities between stimuli (reading material) and thus assimilate them, and also would be able to determine when new schemata are needed for adequate accommodation of a surplus of categories or schemata. Therefore, we can see how well the theories of schemata, assimilation, accommodation and equilibration and reading comprehension are interrelated with each other.

According to Woolfolk \& Winne and Perry (2003) Piaget has taught us that we can learn a great deal about children's thinking by listening carefully and paying close attention to their ways of solving their problems and encountering with new situations. By understanding children's or students thinking, we can better match teaching material to the children's and student's current abilities. Schemata have another utility for curriculum designers, administrators, teachers and researchers in the realm of second language acquisition, especially reading and listening. 
For example, curriculum or course book designers by using need analysis technique, can include a variety of topics for students reading comprehension related to their existing schemata. Teachers when they teach a reading lesson can make a warm up by advance organizers and relate the incoming stimuli to the students existing schemata.

\section{Matching Strategies to Abilities}

An important implication of Piaget's theory for teaching is what J.M. Hunt years ago (1961, as cited in Woolfolk, Winne and Perry 2003) called "the problem of the match" Students must be neither bored by work that is too simple nor left behind by teaching they cannot understand. According to Hunt, disequilibrium must be kept "just right" to encourage growth. Setting up reading and listening activities that would lead students the error can generate disequilibrium. "When students experience some conflict between what they think should happen (a piece of wood should sink because it is big) and what actually happens (it floats), they may rethink their understanding, and new knowledge may develop" (Woolfolk, Winne \& Perry, 2003, p. 38).

To evaluate their thinking in the process of equilibration and construction of meaning in a reading or listening activity, students need to interact with their teachers and peers to challenge their existing schemata, to receive feedback and to watch how other students work out the problems. In these situations, disequilibrium is often set in motion naturally when the teacher or another student suggests the new way of solving the problem. Concrete experiences provide the raw material fir thinking. Communication and interaction make students use, evaluate and finally adapt their current thinking abilities. (Woolfolk, Winne \& Perry, 2003). In other words, interaction and communication in reading classes with fellow students help students to reach to the cognitive equilibrium and show them how to avoid from cognitive disequilibrium and finally how to adapt their cognitive development.

\section{E. Interlanguage and Equilibration}

The process of interlanguage is closely related to the process of equilibration. As Williams\& Burden (2000) pointed out, when we receive input of a language, for example, when we read a novel, we need to modify what we already know about the language by the process of accommodation, so as to fit the new information (reading material) into our existing knowledge by the process of assimilation. By these processes, we gradually develop our knowledge of how the system of new language operates. In other words, we develop our interlanguage. Interlanguage in the field of second language acquisition is defined as learner's knowledge of target language that is gradually re-shaped as it more closely approximate to that language by the process of assimilation, accommodation and finally equilibration (p. 23). So we can infer that if we read more and more material in the target language, our interlanguage by the process of assimilation and accommodation can be developed and approximated to the target language.

\section{F. Critical Thinking, Reading Comprehension and the Process of Equilibration}

The literature indicates that there is no consensus regarding the definition of critical thinking. A multiplicity and variation of definitions of critical thinking are reflective of the way in which educators and scholars define it (Aloqaili, 2001; Minter, 2010 as cited in Aloqaili, A. S. 2011). Romeo (2010 as cited in Aloqaili, A. S. 2011) explains that there is currently a lack of an accepted framework for critical thinking, so that there is not a widely acknowledged and accepted theoretical definition. Some educators and psychologists deal with critical thinking as a narrow concept, whereas others view critical thinking as a broad concept. For example, Beyer (1987 as cited in Aloqaili, A. S. 2011) defined critical thinking in a narrow sense as convergent thinking. He accounts for convergent critical thinking, in contrast to creative thinking which is divergent.

Beyer (1985 as cited in Aloqaili, A. S. 2011) has argued that critical thinking is not a process a unified operation consisting of a number of operations through which one proceeds in sequence. Mcpeck (1981 as cited in Aloqaili, A. S. 2011) has offered this broad definition for critical thinking, the propensity and skill to engage in an activity with reflective skepticism. Ennis (1962 as cited in Aloqaili, A. S. 2011) has dealt with critical thinking with a narrow sense. He stated that critical thinking is "the correct assessing of statements.

Another scholar who has provided a broad definition for critical thinking is Facione (1984 as cited in Aloqaili, A. S. 2011) who developed a definition of critical thinking that incorporates evaluation and problem solving. Facione indicates that it is possible to evaluate critical thinking by evaluating the adequacy of the arguments that express that thinking. He stated that critical thinking is the development and evaluation of arguments. According to Facione (1984) constructing arguments include the usual steps of problem solving which are: (1) determining background knowledge, (2) generating initially plausible hypotheses, (3) developing procedures to test these hypotheses, (4) articulating an argument from the results of these testing procedures, (5) evaluating the arguments, and (6), where appropriate, revising the initial hypotheses.

The relationship between critical thinking and reading is well established in the literature. For example, Norris and Phillips (1987 as cited in Aloqaili, A. S. 2011) point out that reading is more than just saying what is on the page; it is thinking. Moreover, Beck (1989 as cited in Aloqaili, A. S. 2011) asserts there is no reading without reasoning. Also, among those researchers and theoreticians who recognize that reading involves thinking is Ruggiero (1984 as cited in Aloqaili, A. S. 2011). He indicates that reading is reasoning. Yu-hui et al. (2010 as cited in Aloqaili, A. S. 2011) stated clearly that reading is a thinking process to construct meaning. 
Utilizing and combining schema theory with principles of critical thinking are one of the effective ways of enhancing the concept of reading comprehension (Norris and Phillips, 1987 as cited in Aloqaili, A. S. 2011). Schema theory provides powerful rationales for making links between students' individual backgrounds, specific subject area knowledge, and critical thinking (Marzano et al., 1988; Aloqaili, 2005 as cited in Aloqaili, A. S. 2011). According to Anderson (1994 as cited in Aloqaili, A. S. 2011), there are six ways in which schemata function in thinking and in remembering text information. These six ways are:

(1) Most new knowledge is gained by assimilating new information into existing structure; therefore, subject matter learning should build on prior knowledge whenever possible.

(2) The students' existing schemata help to allocate attention by focusing on what is pertinent and important in newly presented materials.

(3) Schemata allow and direct the inferential elaboration of incoming information and experience.

(4) Schemata allow orderly searches of memory by providing learners with a guide to the types of information that should be recalled.

(5) Schemata facilitate the thinking skills of summarizing and editing.

(6) Schemata permit inferential reconstruction when there are gaps in memory, which means that they help the learner generate hypotheses about missing information.

The above discussions and claims show that there is a close relationship between schemata, critical thinking and comprehension processing. Prior knowledge operates as a bridge in establishing connections between thinking critically and text information processing. This connection finally makes the critical text comprehension possible. When this connection is established, the process of equilibrium can be facilitated, in other words, critical thinking by using schemata facilitate the processes of assimilation and accommodation and avoid the process of disequilibrium.

Critical thinking is also related to whole word approach. This approach is concerned with teaching and learning reading and assumes that the whole meaning goes beyond each of the words in the sentences. Critical thinking plays a significant role in this approach as a mechanism or strategy which students can rely on to comprehend text critically. Critical thinking in this approach is based on student's schemata and their speculations. In this approach students based on their own ideas and interpretations and schematic knowledge make sense of the text and make the processes of assimilation, accommodation and in the final run equilibrium possible.

\section{G. Neo-Piagetian View of Equilibration and Reading Comprehension}

As we saw, Rutherford in his modern view of accommodation and assimilation added a new dimension of sociocultural realms which is concerned with social, cultural, and contextual factors that a person need in the process of equilibration culturally. So we can say that, although cognitive development is a necessity and prerequisite, contextual and socio cultural factors also play a paramount role in cognitive development too. Now days no person can neglect the implications and influences of socio cultural and contextual factors on second language acquisition. As far as reading comprehension is concerned, these factors play an important role in bottom up processing. The main reason is that, there are various genres with special characteristics related to the contextual and socio-cultural dimensions and factors. If a student wants to read a genre that is about England literature, he can comprehend the text well, provided that he is familiar with the contextual and socio- cultural characteristics of the text. In this situation, he can assimilate and accommodate the incoming information culturally and makes the cultural equilibrium possible. So these conditions generally facilitate the process of cognitive equilibrium, avoid cognitive disequilibrium and in a final run, make the overall cognitive development possible.

\section{CONCLUSION}

The literature reveals the significant importance of equilibration in Piaget's theory of cognitive development. Equilibration by two processes, namely; assimilation and accommodation leads the child or learner to the cognitive adaptation and makes the cognitive development possible. By incorporating Piaget's construct of equilibrium into educational contexts, (e.g. language learning and teaching), it has been indicated that there is a strong relationship between listening and reading comprehension and the process of equilibrium. In this relationship schemata play an important role and make a process of assimilation and accommodation possible and avoid the reader or listener from disequilibrium and lead him or her to the cognitive adaptation which is a necessary condition for cognitive development. The schemata also play a role as a bridge between individual backgrounds, specific subject area knowledge, and critical thinking and so, make a link between critical thinking and reading comprehension and lead the reader to the process of critical reading comprehension. In this process reader's schemata provides powerful rational for the processes of assimilation, accommodation and finally equilibration which lead the reader to comprehend the text critically. It has been also shown that Rutherford's neo-Piagetian view of assimilation and accommodation has influenced the process of equilibration culturally by taking into account the socio-cultural dimensions which now days have significance importance in the realm of language teaching and learning. Finally, it is worth noting that, in the realm of psychology, if a theory can't be implicated into settings such as educational institutions for various practical reasons, it may lose its values. But research and evidences have shown that, the theory under investigation have had its practical influences and 
impacts on many educational settings specially language learning and teaching and even other settings and contexts. So, this theory has saved its utilities and values since decades ago because of these reasons.

\section{REFERENCES}

[1] Aloqaili, A. S. (2012). The relationship between reading comprehension and critical thinking: A theoretical study. Journal of King Saud University-Languages and Translation, 24(1), 35-41.

[2] Armstrong, K. H., et al. (2014). Early child development theories. Evidence-Based Interventions for Children with Challenging Behavior. Springer Science Business Media New York, 22-28.

[3] Cook, J. L., \& Cook, G. (2005). Child development. London: Pearson education: Allyn \& Bacon.

[4] Fajemidagra, o. (n.d.). Piaget's construct of equilibration: its role in cognitive development and its implications for mathematics/science instruction in Nigerian secondary schools.

[5] Grabe, W. (1988). Reassessing the term 'interactive'. In Interactive approaches to second language reading, Patricia L. Carrell, Joanne Devine, and David E. Eskey (eds.), 56-70. Cambridge: Cambridge University Press.

[6] Lynch, T. (2006). Academic listening: Marrying top and bottom. Current Trends in the Development and teaching of the four language skills 12(5), 91-101.

[7] Pitchard, A., \& Wollard, J (2010). Psychology for the classroom: constructivism and social learning (1th ed.). Oxford: Routledge: Taylor \& Francis.

[8] Rutherford, D. G. (2011). A Model of Assimilation and Accommodation in the Cognitive \& Cultural Realms. Dynamical Psychology. Retrieved from: http://dynapsyc.org/2011/Rutherford_2011.pdf.

[9] Swaffar, J. K. (1988). Readers, texts, and second languages: The interactive processes. The Modern Language Journal, 72(2), 123-149.

[10] Williams, M., \& Burden, R. L. (2000). Psychology for language teachers (3th ed.). Cambridge: Cambridge University Press.

[11] Woolfolk, A. E., Winne, P. H., \& Perry, N. E. (2003). Educational psychology (2th ed.). Pearson Education Canada Inc: Allyn $\&$ Bacon, Inc Needham Height, MA.

[12] Yang, Y. F. (2010). Cognitive conflicts and resolutions in online text Revisions: three profiles. Educational Technology and Society, 13 (4), 202-214.

Hamidreza Babee Bormanaki is a graduate student of TESOL in the Department of English Language and Literature at the University of Guilan, Rasht, Iran. He has examined Construct Validity in the IELTS Listening Test as part of his master's thesis.

Yasin Khoshhal is a graduate student of TESOL in the Department of English Language and Literature at the University of Guilan, Rasht, Iran. His research interests are ESP, syllabus design and material development, and new trends in teaching the language skills. He has published articles in International Journal of Pedagogies and Learning, and other accredited journals. 